\title{
Trichorhinophalangeal syndrome type 2
}

INSERM

\section{Source}

INSERM. (1999). Orphanet: an online rare disease and orphan drug data base.

Trichorhinophalangeal syndrome type 2. ORPHA:502

Langer-Giedon syndrome, also known as trichorhinophalangeal syndrome type 2, is a very rare, genetic, multiple cong enital anomaly disorder characterized by bone abnormalities, distinctive facial features, multiple exostoses, and intellectual disability. 